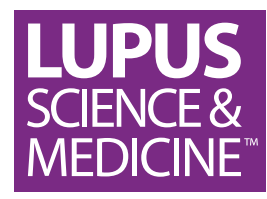

\title{
Comorbidity and healthcare utilisation in persons with incident systemic lupus erythematosus followed for 3 years after diagnosis: analysis of a claims data cohort
}

Katinka Albrecht (D) , ${ }^{1}$ Imke Redeker, ${ }^{1}$ Martin Aringer, ${ }^{2}$ Ursula Marschall, ${ }^{3}$ Anja Strangfeld, ${ }^{1}$ Johanna Callhoff (i) ${ }^{1,4}$

To cite: Albrecht K, Redeker I, Aringer $\mathrm{M}$, et al. Comorbidity and healthcare utilisation in persons with incident systemic lupus erythematosus followed for 3 years after diagnosis: analysis of a claims data cohort. Lupus Science \& Medicine 2021;8:e000526. doi:10.1136/ lupus-2021-000526

\section{- Additional supplemental} material is published online only. To view, please visit the journal online (http://dx.doi.org/10. 1136/lupus-2021-000526).

Received 9 June 2021 Accepted 13 August 2021

Check for updates

(c) Author(s) (or their employer(s)) 2021. Re-use permitted under CC BY-NC. No commercial re-use. See rights and permissions. Published by BMJ.

For numbered affiliations see end of article.

Correspondence to

Dr Katinka Albrecht; albrecht@ drfz.de

\section{ABSTRACT}

Objective To analyse comorbidity and healthcare utilisation in individuals with SLE.

Methods A cohort of individuals with incident SLE diagnosis in 2016 were investigated using claims data from a German statutory health insurance fund. Concomitant diagnoses, medical prescriptions, hospitalisation and sick leave were analysed in the year prior to diagnosis and during a 3-year follow-up in comparison with age-matched and sex-matched controls (1) without autoimmune diseases and (2) with incident diabetes mellitus. Sensitivity analyses were performed excluding cases with additional autoimmune diagnoses and without prescription of antimalarials.

Results Among 571 individuals with SLE, hypertension $(48 \%)$, depression $(30 \%)$, hyperlipidaemia $(25 \%)$, osteoarthritis (25\%) and osteoporosis (20\%) were the most frequent comorbidities in 2016. Cerebrovascular disease was documented in $9.6 \%$. The number of drugs (mean 9.6, $\Delta+6.2)$, hospitalisation $(40 \%, \Delta+27 \%)$ and days on sick leave (median 46 days, $\Delta+27$ days) increased significantly in the first year with SLE diagnosis. Individuals with SLE were more frequently hospitalised and had more medications compared with both control groups (all $p<0.001)$. The increase in comorbidity diagnoses was low in controls without autoimmune diseases, while controls with diabetes showed a more pronounced increase in cardiovascular risk factors, but less in osteoporosis and cerebrovascular disease. Sensitivity analyses showed comparable results.

Conclusion Comorbidities are frequently detected at the time of diagnosis of SLE. High numbers of drug prescriptions and hospitalisation following SLE diagnosis reflect the comprehensive disease burden. The comparison with incident diabetes shows that differences with controls without autoimmune disease are overestimated by detection bias.

\section{INTRODUCTION}

SLE is a systemic autoimmune disease which by nature is prone to lead to manifold organ involvement. Comorbidity in SLE is common,

\section{Key messages}

What is already known about this subject?

- Patients with SLE have a high burden of comorbidity.

What does this study add?

- Numerous comorbidities are frequently detected at the time of SLE diagnosis.

- Increasing rates of medical prescriptions, hospitalisation and sick leave demonstrate comprehensive disease burden in the first 2 years after SLE onset.

- Cerebrovascular disease was diagnosed in every tenth individual at the time of lupus diagnosis.

How might this impact on clinical practice or future developments?

- Comorbidity assessment is essential already at the onset of SLE.

Detection bias needs to be considered when comparing data of persons with chronic diseases with controls using claims data.

occurring as a consequence of chronic inflammation, organ damage, anti-inflammatory therapy and psychosocial effects. Data from the UK indicate a great burden of comorbidity in people with SLE compared with population controls. ${ }^{1}$ An increased risk of numerous comorbidities is already present prior to diagnosis but also remains in the further course of the disease. ${ }^{2}$ The European League Against Rheumatism (EULAR) recommendations for the management of SLE address the importance of assessing cardiovascular and infectious risk factors in SLE. ${ }^{3}$ In addition, bone health is considered a major comorbidity target. ${ }^{45}$ So far, there have been only limited data on comorbidities in people with SLE in Germany. Patients with SLE who are treated in specialised arthritis centres are documented in the German 
National Database ${ }^{6}$; however, majority of patients have long-standing disease and numbers on incident SLE cases are small. ${ }^{78}$ The German LuLa study, the second relevant cohort in Germany, focuses on self-reported outcomes instead. ${ }^{9}$ A recent claims data analysis from a German health insurance fund database has identified a rising incidence of SLE diagnoses accompanied by an increase in healthcare resource utilisation and costs. ${ }^{10}$ The approach for this study was to use health insurance data to approximate the frequencies of comorbidity diagnoses in SLE in comparison with population-related controls. ${ }^{11}$ SLE and comorbidity-related drug prescriptions, sick leave and hospitalisation were examined in individuals with incident SLE diagnosis in the year prior to diagnosis and during a 3-year follow-up in comparison with age-matched and sex-matched controls.

\section{PATIENTS AND METHODS}

\section{Definition of SLE diagnosis in claims data}

A cohort of persons with incident SLE diagnosis was created using data from a large nationwide statutory health insurance fund (BARMER). In Germany, around $90 \%$ of the population are insured in one of the statutory health insurance companies. The other $10 \%$ are insured in private health insurance companies. The BARMER data cover around $11 \%$ of the German population from all areas of Germany and are representative of the German population in terms of socioeconomic status. There are more women older than 50 than in the general population and less men younger than 50 . Insurance fees between the different German statutory health insurances differ only marginally.

Individuals aged $\geq 18$ years were identified based on SLE diagnosis (M32.1: SLE with organ or systemic involvement; M32.8: other forms of SLE; M32.9: SLE, unspecified), ${ }^{12}$ according to the German modification of the International Statistical Classification of Diseases (ICD-10). To identify incident cases, two outpatient or one inpatient diagnosis was required to be present in 2016, 2017 and 2018 but not in the 2 previous years (2014 and 2015). This cohort was analysed in the year prior to diagnosis (2015), the index year (2016) and for 2 consecutive years (2017 and 2018). Persons with SLE diagnoses recorded in 2014 and 2015 and persons who were not continuously insured from 2014 to 2018 or did not have an SLE diagnosis in at least two quarters in 2017 and/or 2018 were excluded (see flow chart in figure 1). Persons with druginduced SLE (M32.0; $\mathrm{n}=83$ in 2016) were also excluded from the analysis. To reduce misclassification related to sporadic diagnoses, we selected a stricter requirement of at least two ICD-10 diagnoses in each of the years from 2016 until 2018 than usually performed. ${ }^{10-12}$

We performed a sensitivity analysis of the diagnosis validation. We further analysed the accuracy of identifying patients with SLE in the claims database following the validation algorithm of Schwarting et al, ${ }^{10}$ which includes primary diagnosis in hospital, outpatient diagnosis by a specialised physician, performance of laboratory ANA tests, antimalarial or immunosuppressive medication, or organ involvement in any of the years 2016, 2017 or 2018.

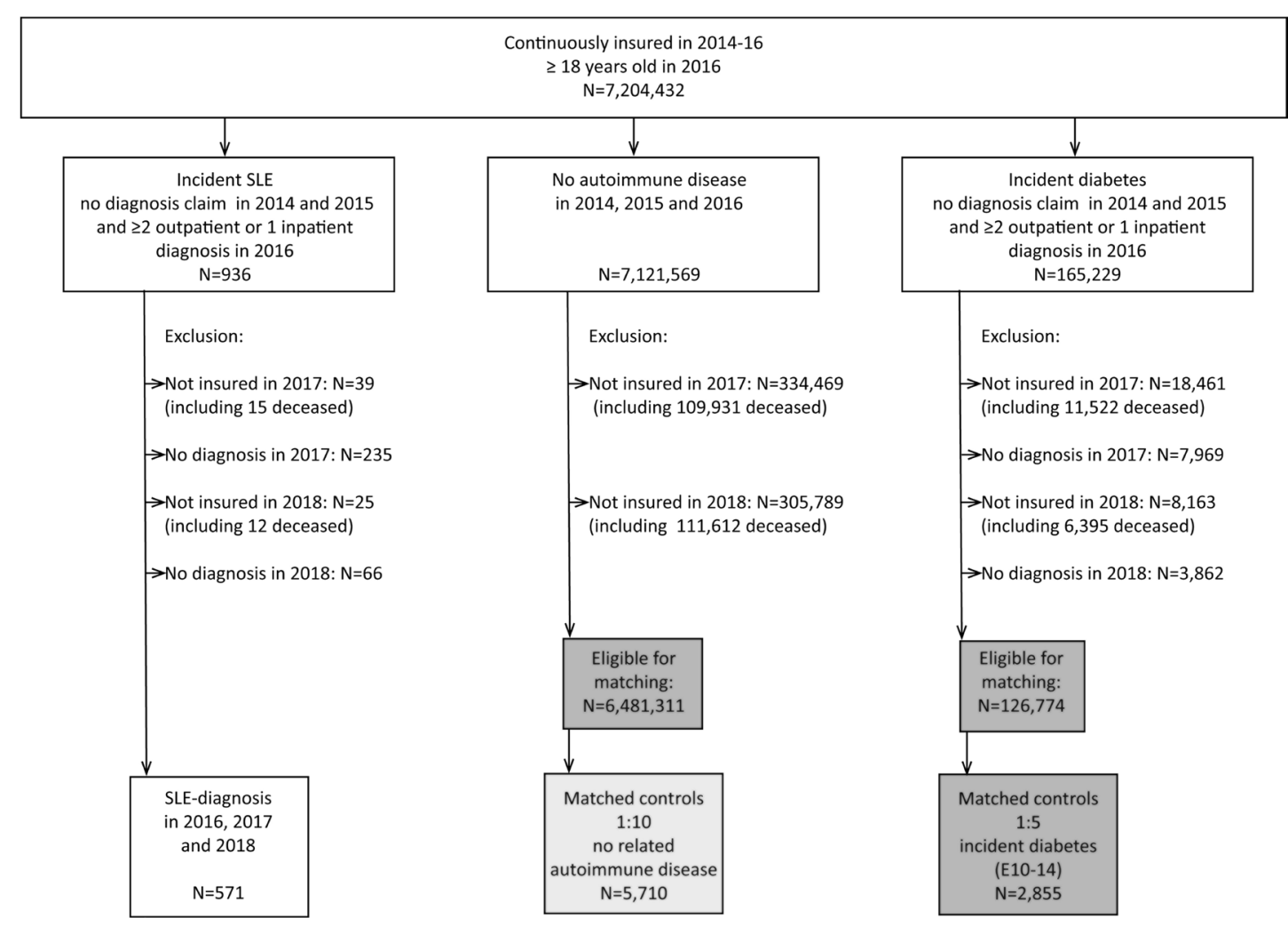

Figure 1 Flow chart. 


\section{Characterisation of the SLE cohort}

The mean age and proportion of women were calculated in the index year. Rheumatology care was identified by the medical specialist number and by identifying those physicians who used claims codes exclusive to rheumatologists. All patients who visited a rheumatologist at least once in the corresponding year were considered to be rheumatologically treated. Diagnostic ANA testing was analysed using the claims codes from the German evidence-based medicine catalogue. Organ manifestations that are specifically assigned or related to SLE were identified by ICD-10 codes. The codes can be referenced in online supplemental table 1: glomerulonephritis, pericarditis, endocarditis, cerebral arteritis, dementia, encephalitis, polyneuropathy, myopathy, lung involvement, Raynaud's syndrome and antiphospholipid syndrome. Anti-inflammatory therapies were identified via the Anatomical Therapeutic Chemical Classification (ATC). ATC codes are shown in online supplemental table 1: glucocorticoids, (hydroxy) chloroquine, azathioprine, methotrexate, mycophenolate mofetil, ciclosporin, leflunomide, belimumab, rituximab and abatacept. For biologics and cyclophosphamide, procedure codes for administration in hospital were also considered.

\section{Comparison of outcomes derived from claims data}

Comorbidity diagnoses were identified by ICD-10 codes. The codes are shown in online supplemental table 1: hypertension, hyperlipidaemia, obesity, cerebrovascular diseases, thrombosis, renal disease, chronic obstructive pulmonary disease, hypothyroidism, osteoarthritis, osteoporosis (total, with and without pathological fracture), fibromyalgia, depression, polyneuropathy, solid tumour and metastatic cancer. Where applicable, definitions from the Elixhauser comorbidity score were used, and the Elixhauser score was calculated. ${ }^{12}$ Specialist care on comorbidities was identified by medical specialist numbers. Lipid profile testing was used to detect differences in the frequency of comorbidity screening and was identified from the German evidence-based medicine catalogue.

Using inpatient data from the hospital claims data, persons were counted as being hospitalised in the corresponding year if they had at least one inpatient admission. Sick leave and days of sick leave were assessed for each corresponding year. For sick leave, only persons $<65$ years (corresponding to the German retirement age applicable for the years analysed) were included. Since the occupational status was not available, the average duration was calculated and reported in median days instead of the proportion of persons with sick leave. To count the days on sick leave for the corresponding year, sick leave periods starting before 1 January or ending after 31 December were cropped so that the maximal possible number of days on sick leave was 365 days.

The mean number of prescribed medications including SLE anti-inflammatory therapy was calculated. Medical prescriptions related to comorbidity were identified by ATC codes (the codes are listed in online supplemental table 1: cardiovascular therapy (antihypertensives, beta-blockers, diuretics), lipid-lowering therapies, osteoporosis therapies, antidepressants, non-steroidal antirheumatic drugs (NSAIDs), other analgesics and opioids. As prescriptions are not assigned to a specific diagnosis, symptomatic pain medications including NSAIDs were listed here and not as lupus-specific therapy.

The data cover all medications for which costs are payed by the health insurance. This generally includes all medications we analysed in this manuscript, and also those that could be bought over the counter (such as ibuprofen), but were prescribed by a physician. Copayments are generally small, and for people with a chronic illness such as SLE there is a limit of $1 \%$ of the patient's gross income per year. After a person has reached this limit, there are no copayments anymore.

\section{Statistical analysis}

Control groups

To compare the frequency of comorbidity claims, drug prescriptions, sick leaves and hospitalisations, a control group was randomly selected and matched 10:1 for age and sex from the insurance population without SLE and without any of the following rheumatic diseases: rheumatoid arthritis (M05, M06), myositis (M33), systemic sclerosis (M34), Sjögren's syndrome (M35.0) and mixed connective tissue disease (M35.1). To account for a possible detection bias in persons with incident diagnoses compared with controls without, a second control group with an incident diagnosis of diabetes (ICD-10: E10-E14) was randomly selected and matched 5:1 for age and sex to the SLE cohort. Diabetes was selected for comparison in the expectation that comparable comorbidity screening is performed at the time of diagnosis, and with regard to cardiovascular risk factors in particular. Persons in the control groups also had to be continuously insured between 2014 and 2018 (figure 1). In the diabetes control group, diagnostic codes of diabetes had to be documented in at least two quarters of each year (2016, 2017 and 2018), but no codes were allowed to be present in 2014 and 2015, analogue to the SLE cohort.

The frequencies of comorbidities in 2016 were compared with $\chi^{2}$ test or Fisher's exact test, as appropriate. Elixhauser scores and the number of prescribed medications were compared with Wilcoxon-Mann-Whitney-tests.

To exclude cases with uncertainty in SLE diagnosis, two sensitivity analyses were performed excluding (1) cases with additional diagnosis of Sjögren's syndrome, systemic sclerosis, mixed connective tissue disease or tubulointerstitial kidney disease and (2) without prescription of hydroxychloroquine (HCQ), considering that HCQ is recommended as standard therapy for SLE.

\section{Patient and public involvement}

Within the framework of the Targeted Risk Management in Muskuloskeletal Diseases (TARISMA) research project, patient partners were involved in the reporting of our research. 


\section{RESULTS}

\section{Characteristics of individuals with incident SLE}

Out of 7204432 persons $\geq 18$ years who had been continuously insured from 2014 to 2016, a total of 571 persons had incident SLE diagnosis in 2016 and a prevalent SLE diagnosis in 2017 and 2018 (figure 1). A total of 159 persons had an inpatient discharge diagnosis. Outpatient SLE diagnosis was documented by a specialised physician in 325 persons. Between 2015 and 2018, ANA tests were coded in 360, 457 received antimalarial or immunosuppressive medication, 86 had ICD diagnosis of organ involvement, and $320(56 \%)$ were seen by a rheumatologist. Altogether, 519 of the 571 incident SLE cases in this analysis $(90.9 \%)$ fulfilled at least one Schwarting validity criterion.

The mean age in the year of SLE diagnosis was 55.1 years ( $6 \% 18-30$ years, $32 \%$ 31-50 years, $46 \% 51-70$ years, $16 \%>70$ years) and $87 \%$ were female. SLE-associated organ involvement was rarely documented, with the most frequent ICDs being antiphospholipid syndrome (7\%) and kidney involvement $(6 \%)$.

\section{Drug prescriptions related to SLE diagnosis}

Glucocorticoids were prescribed to $55 \%$ of all individuals with SLE in the first year after diagnosis (figure 2). Three in four persons $(75 \%)$ of the incident SLE cohort did not have any prescription of glucocorticoids between 2005 and 2015. Antimalarials (49\%), azathioprine $(13 \%)$, methotrexate $(11 \%)$ and mycophenolate $(8 \%)$ were started in the index year or year 1 , while rituximab $(1.4 \%)$ was mainly introduced in year 1 , and belimumab $(3.5 \%)$ in year 1 and year 2 after diagnosis (figure 2 ). Seven persons $(1.2 \%)$ received cyclophosphamide in the index year.

\section{Comorbidity diagnoses}

Among individuals with SLE, the mean Elixhauser comorbidity score increased from 1.1 in 2015 to 4.5 in the year of diagnosis. Hypertension (48\%), depression (30\%), hyperlipidaemia (25\%), osteoarthritis $(25 \%)$ and osteoporosis $(20 \%)$ were the most frequent comorbidity diagnoses in 2016. Most of the osteoporosis diagnoses were documented as osteoporosis without pathogenic fracture. The proportion of SLE cases with osteoporotic fracture was much higher than in the controls $(4.7 \%$ in 2016 in SLE vs $1.0 \% / 1.1 \%$ in controls). Cerebrovascular disease was documented in $9.6 \%$ of individuals in the year of the first SLE diagnosis. A relevant increase of comorbidity diagnoses in the index year was present particularly in cardiovascular risk factors, cerebrovascular disease, kidney disease, musculoskeletal disorders, depression, hypothyroidism and solid tumours (table 1). After that, the number of comorbidity diagnoses increased only slightly in year 1 and year 2 after SLE diagnosis.

1. In controls without related autoimmune diseases $(n=5710)$, the number of comorbidity diagnoses only slightly increased in the index year (Elixhauser $\Delta+0.3$ ). All comorbidity diagnoses except metastatic cancer

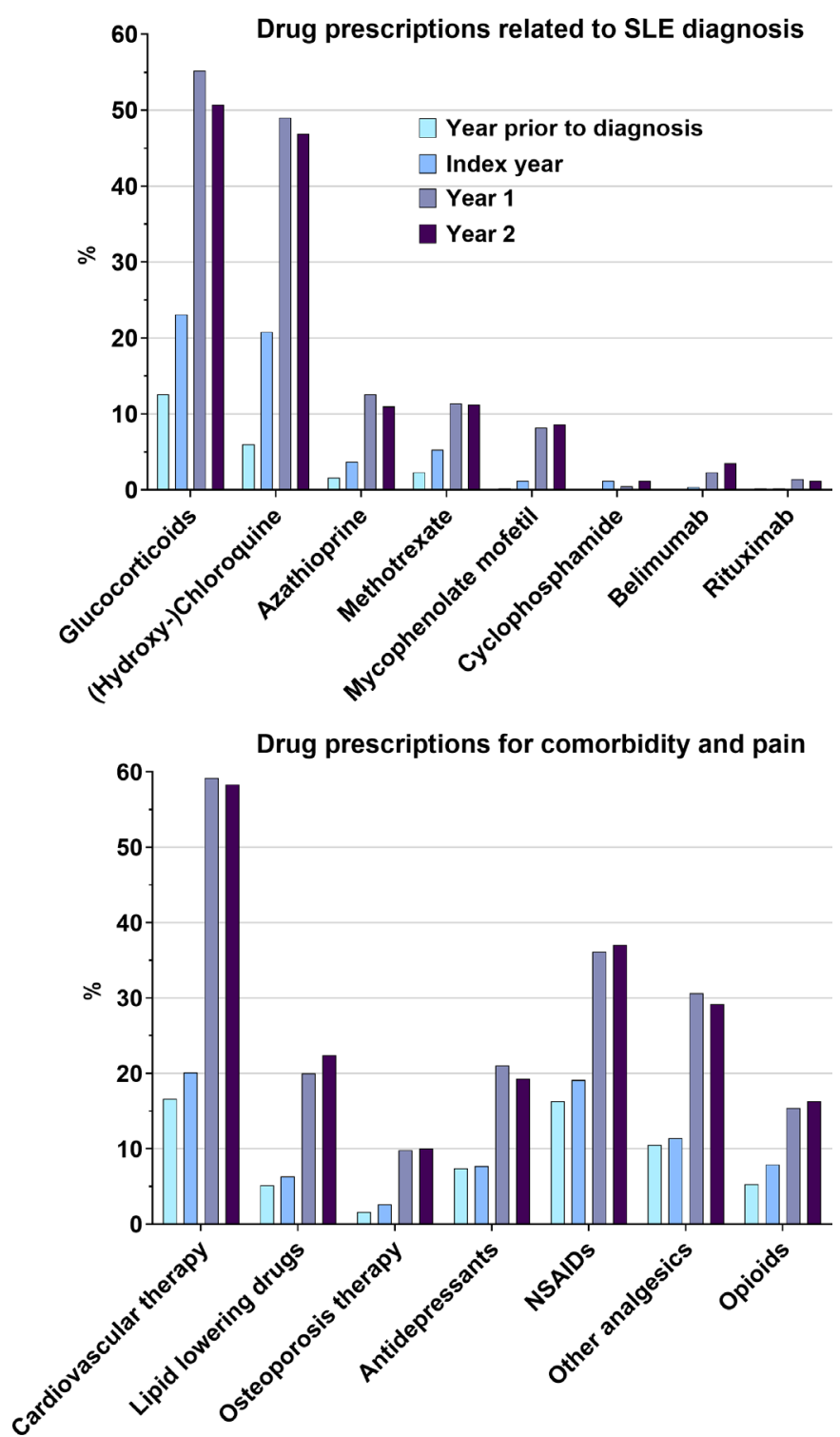

Figure 2 Drug prescriptions in persons with incident SLE in 2016. NSAIDs non-steroidal antirheumatic drugs.

were significantly more frequent in cases with SLE in the index year compared with the controls ( $p$ values are reported in table 1 ).

2. In controls with incident diabetes, cardiovascular risk factors increased to a higher extent and osteoarthritis, osteoporosis, cerebrovascular disease and depression to a lower extent compared with cases with SLE (figure 3). Cerebrovascular disease, thrombosis and kidney disease were significantly more frequent in individuals with SLE compared with controls with diabetes in the index year $($ all $<0.001$; table 1$)$.

\section{Healthcare utilisation}

Diagnostics and specialist care regarding comorbid conditions were significantly more frequent in cases with SLE or diabetes compared with controls without autoimmune disease. In the index year, the lipid profile was tested in $43 \%$ (controls), $60 \%$ (SLE) and $80 \%$ (diabetes). In the study period, nephrology care was visited by $17 \%$ (SLE) 


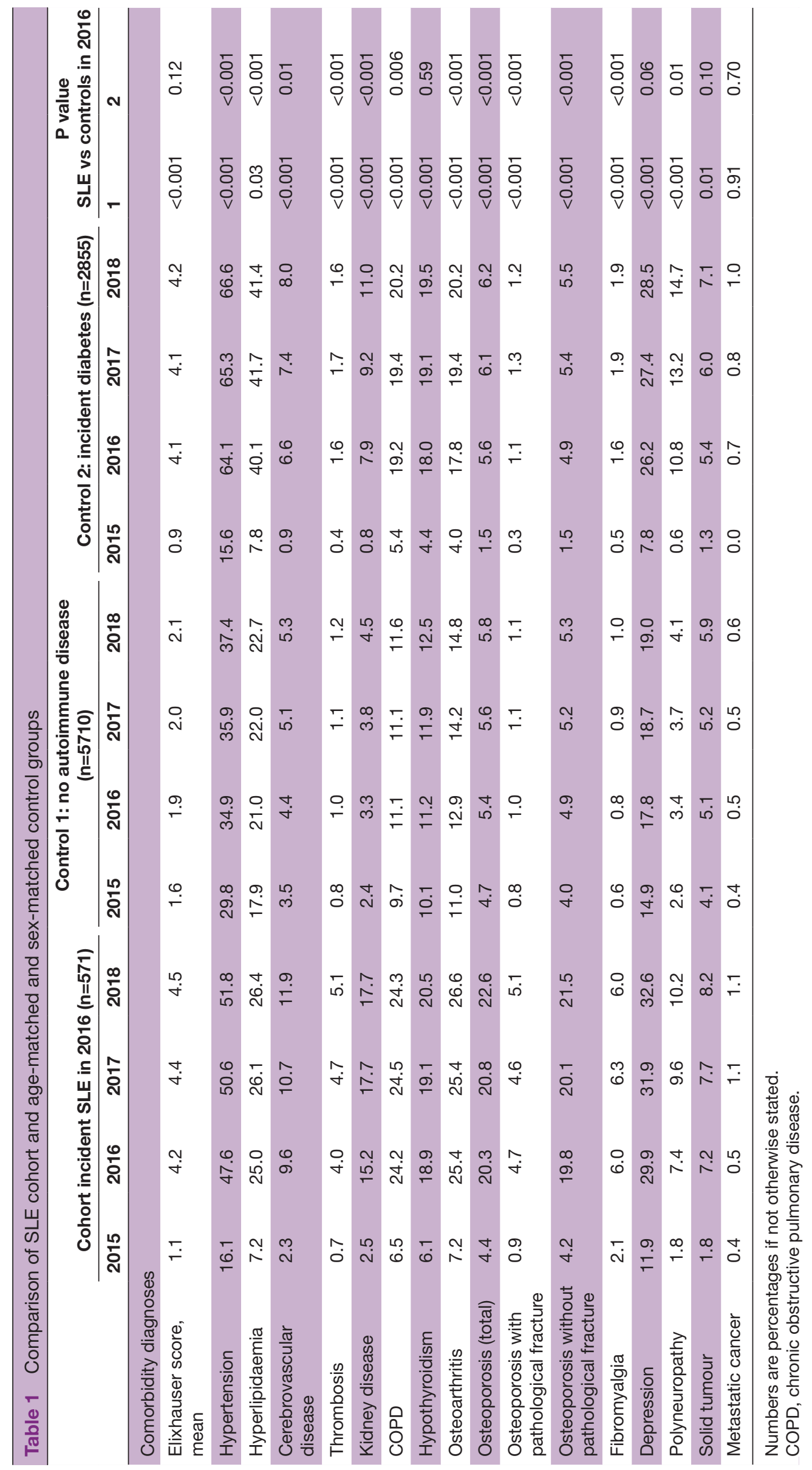


Hypertension

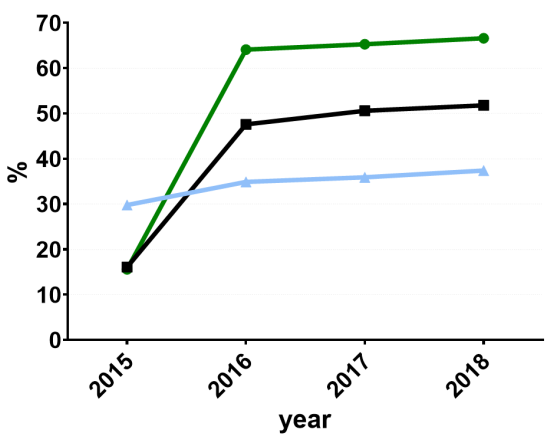

Depression

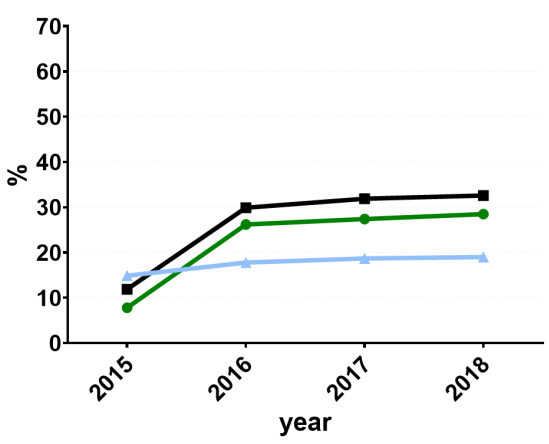

Osteoarthritis

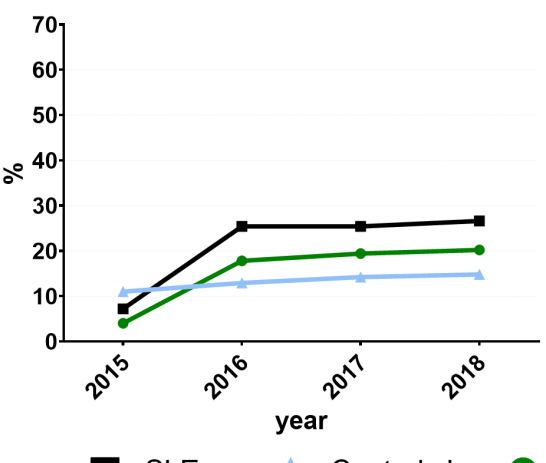

SLE

Controls I
Hyperlipidemia

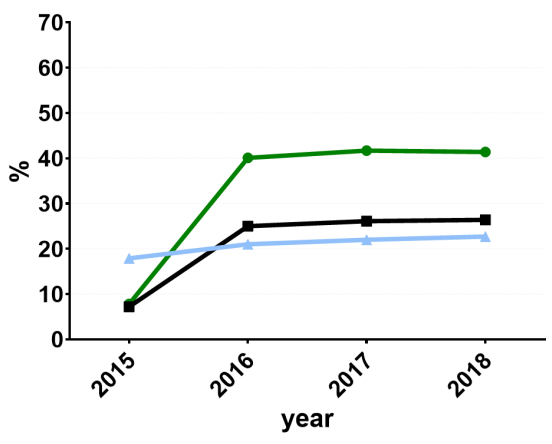

Osteoporosis

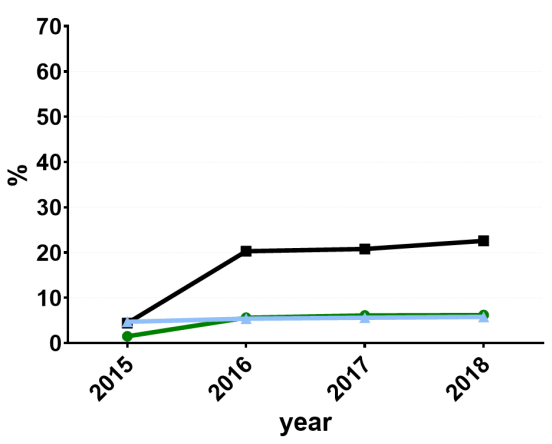

Cerebrovascular disease

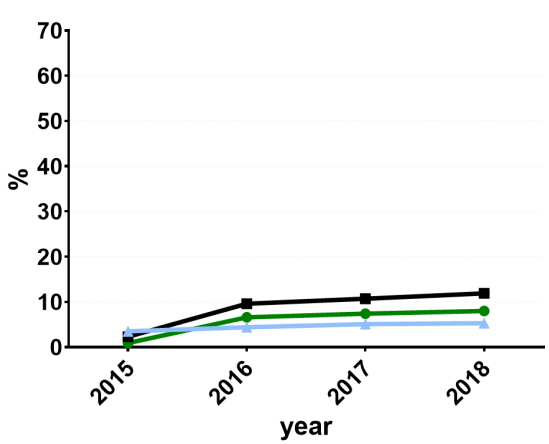

Controls II (diabetes)

Figure 3 Comorbidity recorded in persons with incident SLE in 2016 ( $\mathrm{n}=571, \mathrm{M} 32.1, \mathrm{M} 32.8, \mathrm{M} 32.9)$ and age-matched and sex-matched controls ( $n=5710$ without related autoimmune disease, $n=2855$ with incident diabetes, $E 10-14$ ).

vs $3 \%$ (controls) vs $6 \%$ (diabetes), neurology care by $10 \%$ (SLE) vs $10 \%$ (controls) vs $13 \%$ (diabetes), and a psychiatrist by $1 \%-7 \%$ (all groups).

Hospitalisation increased from $13 \%$ in the year prior to SLE diagnosis to $40 \%$ in the first year after diagnosis. Patients with SLE were significantly more frequently hospitalised compared with both control groups (both $\mathrm{p}<0.001)$. There was no relevant increase in controls without autoimmune disease (from $14 \%$ to $18 \%$ ), while hospitalisation also increased from $7 \%$ to $25 \%$ in patients with diabetes (table 2).

\section{Sick leave}

A total of 430 persons with SLE, 4300 controls without related autoimmune disease and 2150 controls with diabetes were $<65$ years of age. For those who were on sick leave, in the index year the median number of days on sick leave increased compared with the year before diagnosis in cases with SLE (year before diagnosis: 19 days; index year: 46 days) and in controls with diabetes (21 days and 27 days), but not in controls without autoimmune disease (20 days and 14 days).

\section{Drug prescriptions related to comorbidity and pain}

In SLE, the mean number of drug prescriptions increased from 3.4 in 2015 to 9.5 in 2018, exceeding the increase in controls (mean from 3.9 to 5.2) and controls with diabetes (from 2.7 to 7.5$)(\mathrm{p}<0.001)$. Cardiovascular therapies were prescribed to $58 \%$ of persons with SLE, $40 \%$ (controls) and $68 \%$ (diabetes) in the second year after diagnosis. Prescriptions of NSAIDs and opioids roughly doubled after SLE diagnosis, and prescriptions of other analgesics and antidepressants nearly tripled after SLE diagnosis (figure 2). NSAIDs and opioids were prescribed 


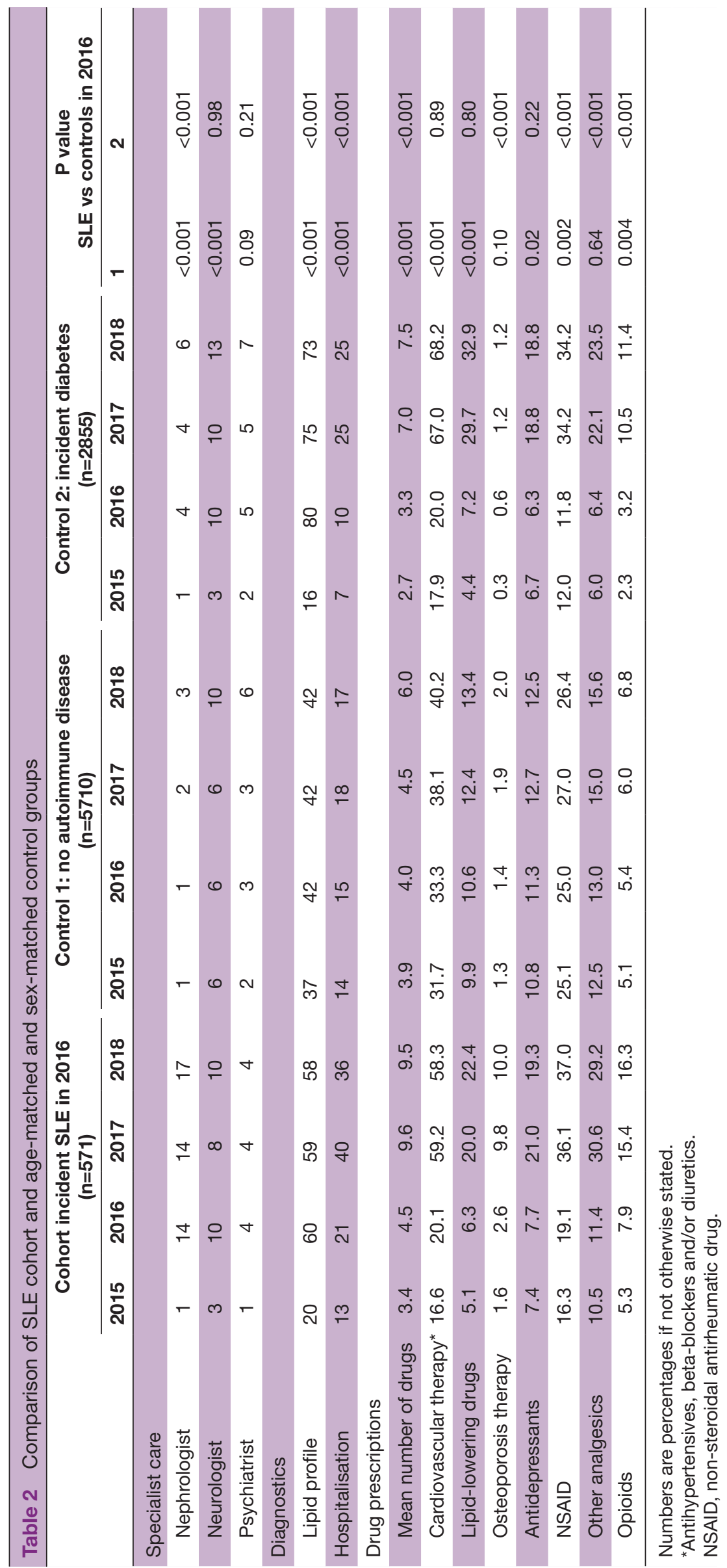

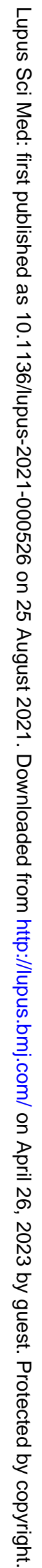


Table 3 Sensitivity analyses of persons without further autoimmune disease and with antimalarial therapy required

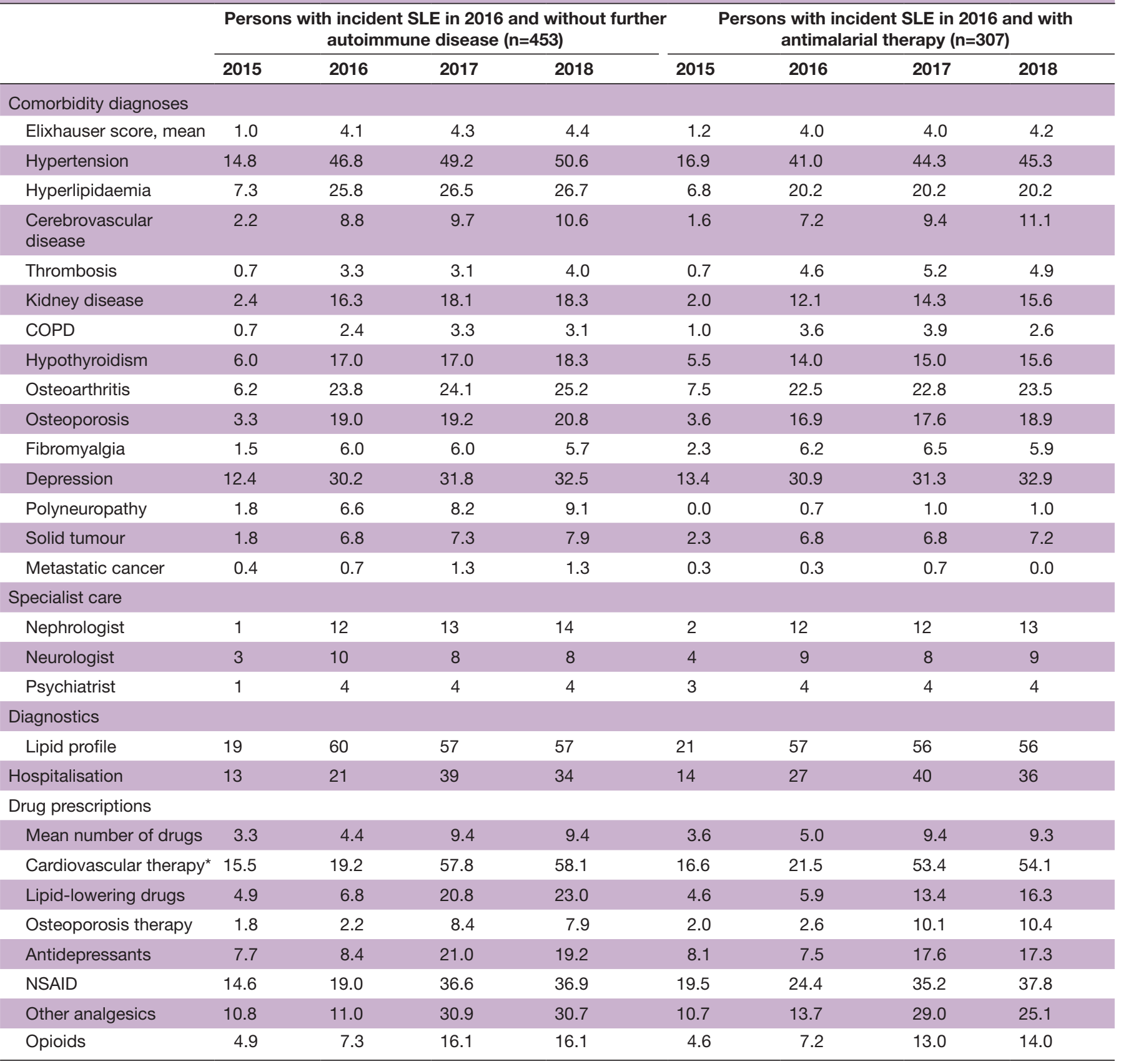

*Antihypertensives, beta-blockers and/or diuretics.

COPD, chronic obstructive pulmonary disease; NSAID, non-steroidal antirheumatic drug.

more frequently in SLE compared with both controls $(\mathrm{p}<0.001$; see table 2$)$.

\section{Sensitivity analyses}

Data from 453 persons without additional autoimmune diagnoses were included in the first sensitivity analysis. The results showed no meaningful differences in the frequencies of comorbidity, drug prescriptions, hospitalisation and sick leave compared with the total sample (table 3).

In the second sensitivity analysis, only data from 312 persons with prescription of HCQ were included. Compared with the total cohort, persons with HCQ were younger (mean age 51.6 years), without a difference in gender distribution $(87 \%)$. Persons with HCQ had slightly less comorbidity (mean Elixhauser 4.0 in the first year after diagnosis). The increase in concomitant diagnoses at the time of SLE diagnosis was comparable with the total cohort (table 3).

\section{DISCUSSION}

This claims data analysis on comorbidity and healthcare utilisation in individuals with SLE revealed a significant increase of comorbidity diagnoses already in the year of the first SLE diagnosis. These results exceeded 
the frequencies in the control group without autoimmune disease, but were, in some aspects, comparable with patients with newly diagnosed diabetes. Increasing medical prescriptions, hospitalisation and days on sick leave were present in the first 2 years after SLE onset. Besides cardiovascular risk factors and renal disease, musculoskeletal and cerebrovascular disease affected a relevant number of persons at SLE onset.

Previous reports have indicated an increased risk of comorbidity in people with SLE, ${ }^{13}$ which is already present in incident disease and affects mortality. ${ }^{2}$ Our study confirms a higher comorbidity burden in comparison with matched general population cohorts. ${ }^{12}$ However, the comparison with a matched cohort with incident diabetes shows a clear detection bias, leading to overestimation of the differences regarding comorbidity diagnoses with healthy controls. It can be assumed that numerous of these comorbidities were already present before SLE or diabetes was diagnosed and are only documented for the first time during the detailed diagnostic process.

Looking at the individual comorbidities, the proportion of depression (every third), osteoporosis (every fifth) and cerebrovascular disease (every tenth individual with SLE) needs to be particularly emphasised. Depression is one of the most frequent mental disorders affecting patients with SLE. ${ }^{14}$ Almost one-third diagnosed with depression at SLE diagnosis point to the frequent mental affection. Increased rates of osteoporosis and fractures have been reported in SLE. ${ }^{15}$ Whereas the regular use of glucocorticoids is likely to be responsible for osteoporosis in longstanding disease, it cannot be causal in incident disease. Pre-existing disease activity and premature menopause are more likely to be reasons for prevalent osteoporosis at SLE onset, ${ }^{45}$ making early screening and risk assessment for fractures essential. It is also possible that the increase in osteoporosis codes for SLE may be due, in part, to heightened evaluation of osteoporosis risk after initiation of glucocorticoids or due to preventative osteoporosis therapies. Osteoarthritis was also more frequently diagnosed in SLE, but it also increased in the diabetes cohort. The increase is likely due to detection bias, as the joints of patients with SLE manifestations are regularly examined, whereas the joints in patients with diabetes are not necessarily examined as a priority. However, incorrect coding of joint manifestations of SLE cannot be ruled out.

Cerebrovascular disease is a known risk factor for mortality in SLE. ${ }^{16}$ In the claims data, the diagnosis is more frequent compared with both control groups and this already applies to the year of SLE diagnosis.

Healthcare utilisation increases abruptly with SLE diagnosis. This can also be observed to a lesser extent in persons with diabetes. In particular, the increase in drug prescriptions, days on sick leave and hospital stays is substantial. While sick leave data are available, other measures of productivity loss such as presenteeism and impairment of unpaid work activities could not be captured in this analysis.
Besides SLE treatments including antimalarials, NSAIDs, corticosteroids and immunosuppressants, analgesics, opioids and drugs for treating comorbidities also contribute to an average prescription of 10 different drugs in the first and second year after diagnosis. US claims data show comparable medication use, frequent inpatient admissions and physician visits leading to high costs in the first postdiagnosis year. ${ }^{17}$ Even if in Germany opioids are not prescribed as commonly as in the USA, where every third patient with SLE received opioids, ${ }^{18}$ the frequency of prescription remains problematic. In addition, other analgesics, NSAIDs and antidepressants are also frequently prescribed.

Antimalarial prescription rates were comparatively low in this study, with $49 \%$ of the patients getting at least one prescription. We can compare this with the percentage receiving antimalarials in the German National Database of the collaborative arthritis centres. In 846 patients with a physician-reported SLE diagnosis in 2016, $64 \%$ received HCQ. ${ }^{6}$ The National Database only covers patients in rheumatological care. In the incident SLE cohort in this manuscript, only $56 \%$ of the patients had contact with a rheumatologist. This might be a reason that antimalarial prescription rates are even lower in these patients.

The cardiovascular risk factors hypertension and hyperlipidaemia were more often present in persons with a diabetes mellitus diagnosis than in SLE, just as for prevalent SLE and diabetes mellitus patients among US Medicaid patients. ${ }^{19}$ This could also be a result of detection bias; for example, lipid profiles were performed $33 \%$ more often in incident diabetes mellitus cases than in incident SLE. Lipid testing was also higher among patients with diabetes mellitus in the US Medicare data. ${ }^{20}$ This could be problematic because another analysis of US Medicaid data shows that the incidence rate ratio for heart failure is comparable among patients with SLE and diabetes mellitus. ${ }^{21}$

The mean age and the proportion of women in this cohort are slightly higher compared with other incident lupus cohorts. ${ }^{210}$ This can be explained by a higher proportion of women and a somewhat older population within the BARMER compared with the average of the statutorily insured population in Germany.

Claims data analyses have several limitations. The greatest limitation remains diagnostic uncertainty due to the lack of clinically validated diagnosis. Previous validation studies for the identification of SLE in administrative and claims databases had a positive predictive value in the range of $70 \%-90 \% .{ }^{22}$ To reduce potentially incorrect diagnostic codes, we included only individuals with continuous SLE diagnosis over 3 years. By adapting the validation algorithm of Schwarting et al, ${ }^{10}$ we were able to classify $91 \%$ of the cases with a validity criterion. We further repeated the analyses only for cases with antimalarial therapy and without additional related autoimmune diagnoses that make SLE less likely. The high agreement between the analyses and the $50 \%$ proportion 
of individuals with antimalarials suggests a good coverage of actual SLE cases.

A further limitation is that the diagnostic measures, for example, ANA tests, cannot be fully determined, as they are often billed at standard rates without specific numbers. In addition, diagnoses are often documented non-specifically, without organ manifestations being specifically reported. Therefore, no robust data on the prevalence of these manifestations can be generated from the data. The strengths of the study are the populationbased sample, the high number of people with incident SLE and the possibility of collecting different matched control groups within the same population.

Regarding the generalisability of our data, the German health insurance data show a good estimate of the actual comorbidity diagnoses and prescription rates, which can be compared with data from other countries. Countryspecific differences in healthcare delivery and uptake need to be taken into account. From a methodological point of view, ICD-10 codes are used ubiquitously and it is becoming increasingly important to interpret health insurance data correctly, as these are being used more and more for research purposes.

\section{CONCLUSIONS}

Comprehensive diagnostic measures after first manifestation of SLE reveal other concomitant diseases that are already present at the time of diagnosis, pointing towards the need for regular comorbidity assessment, already in incident disease. For comparisons between cohorts of patients with incident illness, the control group should be carefully selected. By considering different control groups, the influence of detection bias can at least be estimated to some extent.

\section{Author affiliations}

${ }^{1}$ Epidemiology Unit, German Rheumatism Research Centre, Berlin, Germany ${ }^{2}$ Division of Rheumatology, Department of Medicine III, University Medical Center and Faculty of Medicine Carl Gustav Carus at the TU Dresden, Dresden, Germany ${ }^{3}$ Institute for Health System Research, Barmer, Wuppertal, Germany

${ }^{4}$ Institute for Social Medicine, Epidemiology and Health Economics, Charité Universitätsmedizin, Berlin, Germany

\section{Twitter Johanna Callhoff @callhoffj}

Acknowledgements The authors thank BARMER for providing access to data for this study via their data warehouse. The authors also thank the patient partners in the TARISMA project for dedicating their time to add patient views to this project. Thank you to Dr Elena Garal-Pantaler and Professor Andreas Schwarting for their kind support in providing details on their claims data analysis.

Contributors $\mathrm{JC}$ had full access to all the data in the study and takes responsibility for the integrity of the data and the accuracy of the data analysis. Study concept and design: KA, JC. Acquisition, analysis or interpretation of data: KA, IR, MA, UM, $\mathrm{JC}$. Drafting of the manuscript: KA, JC. Critical revision of the manuscript and approval of the manuscript: KA, IR, MA, UM, AS, JC.

Funding The study was supported by the Wolfgang Schulze Foundation of the German Rheuma-Liga and by the Federal Ministry of Education and Research within the TARISMA network (01EC1902A).

Competing interests None declared.

Patient consent for publication Not required.

Provenance and peer review Not commissioned; externally peer reviewed.
Data availability statement Data may be obtained from a third party and are not publicly available. No additional data are available.

Supplemental material This content has been supplied by the author(s). It has not been vetted by BMJ Publishing Group Limited (BMJ) and may not have been peer-reviewed. Any opinions or recommendations discussed are solely those of the author(s) and are not endorsed by BMJ. BMJ disclaims all liability and responsibility arising from any reliance placed on the content. Where the content includes any translated material, BMJ does not warrant the accuracy and reliability of the translations (including but not limited to local regulations, clinical guidelines, terminology, drug names and drug dosages), and is not responsible for any error and/or omissions arising from translation and adaptation or otherwise.

Open access This is an open access article distributed in accordance with the Creative Commons Attribution Non Commercial (CC BY-NC 4.0) license, which permits others to distribute, remix, adapt, build upon this work non-commercially, and license their derivative works on different terms, provided the original work is properly cited, appropriate credit is given, any changes made indicated, and the use is non-commercial. See: http://creativecommons.org/licenses/by-nc/4.0/.

\section{ORCID iDs}

Katinka Albrecht http://orcid.org/0000-0003-0886-0294

Johanna Callhoff http://orcid.org/0000-0002-3923-2728

\section{REFERENCES}

1 Rees F, Doherty M, Grainge M, et al. Burden of comorbidity in systemic lupus erythematosus in the UK, 1999-2012. Arthritis Care Res 2016;68:819-27.

2 Kuo C-F, Chou I-J, Rees F, et al. Temporal relationships between systemic lupus erythematosus and comorbidities. Rheumatology 2019;58:840-8.

3 Fanouriakis A, Kostopoulou M, Alunno A, et al. 2019 update of the EULAR recommendations for the management of systemic lupus erythematosus. Ann Rheum Dis 2019;78:736-45.

4 Fanouriakis A, Bertsias G, Boumpas DT. Response to: 'Bone health, an often forgotten comorbidity in systemic lupus erythematosus: a comment on the new recommendations' by Orsolini et al. Ann Rheum Dis 2020;79:e151.

5 Orsolini G, Bultink IEM, Adami G, et al. Bone health, an often forgotten comorbidity in systemic lupus erythematosus: a comment on the new recommendations. Ann Rheum Dis 2020;79:e150.

6 Albrecht K, Callhoff J, Zink A. Long-term trends in rheumatology care : Achievements and deficits in 25 years of the German national rheumatology database. Z Rheumatol 2019;78:65-72.

7 Albrecht K, Huscher D, Richter J, et al. Changes in referral, treatment and outcomes in patients with systemic lupus erythematosus in Germany in the 1990s and the 2000s. Lupus Sci Med 2014;1:e000059.

8 Brinks R, Hoyer A, Weber S, et al. Age-specific and sex-specific incidence of systemic lupus erythematosus: an estimate from crosssectional claims data of 2.3 million people in the German statutory health insurance 2002. Lupus Sci Med 2016;3:e000181.

9 Chehab G, Sauer GM, Richter JG, et al. Medical adherence in patients with systemic lupus erythematosus in Germany: predictors and reasons for non-adherence - a cross-sectional analysis of the LuLa-cohort. Lupus 2018;27:1652-60.

10 Schwarting A, Friedel H, Garal-Pantaler E, et al. The burden of systemic lupus erythematosus in Germany: incidence, prevalence, and healthcare resource utilization. Rheumatol Ther 2021;8:375-93.

11 Luque Ramos A, Redeker I, Hoffmann F, et al. Comorbidities in patients with rheumatoid arthritis and their association with patientreported outcomes: results of claims data linked to questionnaire survey. J Rheumatol 2019;46:564-71.

12 Quan H, Sundararajan V, Halfon P, et al. Coding algorithms for defining comorbidities in ICD-9-CM and ICD-10 administrative data. Med Care 2005;43:1130-9.

13 Zucchi D, Elefante E, Calabresi E, et al. One year in review 2019: systemic lupus erythematosus. Clin Exp Rheumatol 2019;37:715-22.

14 Park D-J, Kang J-H, Lee K-E, et al. Association of depression with socioeconomic status, anticardiolipin antibodies, and organ damage in patients with systemic lupus erythematosus: results from the KORNET registry. Clin Exp Rheumatol 2018;36:627-35.

15 Tedeschi SK, Kim SC, Guan H, et al. Comparative fracture risks among United States medicaid enrollees with and those without systemic lupus erythematosus. Arthritis Rheumatol 2019;71:1141-6.

16 Bernatsky S, Clarke A, Gladman DD, et al. Mortality related to cerebrovascular disease in systemic lupus erythematosus. Lupus 2006;15:835-9. 
17 Kariburyo F, Xie L, Sah J, et al. Real-world medication use and economic outcomes in incident systemic lupus erythematosus patients in the United States. J Med Econ 2020;23:1-9.

18 Somers EC, Lee J, Hassett AL, et al. Prescription opioid use in patients with and without systemic lupus erythematosus - michigan lupus epidemiology and surveillance program, 2014-2015. MMWR Morb Mortal Wkly Rep 2019;68:819-24.

19 Barbhaiya M, Feldman $\mathrm{CH}$, Chen SK, et al. Comparative risks of cardiovascular disease in patients with systemic lupus erythematosus, diabetes mellitus, and in general Medicaid recipients. Arthritis Care Res 2020;72:1431-9.
20 Chen SK, Barbhaiya M, Fischer MA, et al. Lipid testing and statin prescriptions among Medicaid recipients with systemic lupus erythematosus or diabetes mellitus and the general Medicaid population. Arthritis Care Res 2019;71:104-15.

21 Chen SK, Barbhaiya M, Fischer MA, et al. Heart failure risk in systemic lupus erythematosus compared to diabetes mellitus and general Medicaid patients. Semin Arthritis Rheum 2019;49:389-95.

22 Moores KG, Sathe NA. A systematic review of validated methods for identifying systemic lupus erythematosus (SLE) using administrative or claims data. Vaccine 2013;31 Suppl 10:K62-73. 